\title{
Incidencia de tumor carcinoide en apendicectomía por apendicitis aguda; 5 años de experiencia en HNERM
}

\author{
José M. Ortiz, Elydia Mujica, Duber Gallardo \\ Instituto Nacional de Biología Andina, Facultad de Medicina, UNMSM, y Hospital Nacional Edgardo Rebagliati Martins, EsSalud.
}

\begin{abstract}
Objetivos: Determinar los casos de tumor carcinoide en pacientes apendicectomizados por apendicitis aguda, en el Hospital Nacional Edgardo Rebagliati Martins, EsSalud.

Diseño: Descriptivo, observacional.

Institución: Instituto Nacional de Biologia Andina, Facultad de Medicina, UNMSM, y Hospital Nacional Edgardo Rebagliati Martins, EsSalud.

Materiales: Se revisó 9452 informes de diagnóstico anatomopatológico de apendicectomías recibidas en el Servicio de Patología Quirúrgica, entre el año 2003 y el 2007.

Intervenciones: Revisión de casos con el diagnóstico anatomopatológico.

Principales medidas de resultados: Incidencia de cáncer de tumor carcinoide y su estratificación por edad y género.

Resultados: De las 9452 apendicectomías por apendicitis aguda realizadas, se registró siete casos de tumor carcinoide, con lo cual se obtuvo una incidencia de $0,07 \%$. Dado el volumen de apendicectomías en el HNERM, se observa que el hallazgo de tumor carcinoide se presenta un caso por año. El 100\% de estos casos correspondió a mujeres y las edades variaron entre 11 y 62 años, con una mediana de 26 años.

Conclusiones: El tumor carcinoide es una neoplasia relativamente rara. Sin embargo, se presenta ocasionalmente, por lo que se recomienda el estudio histopatológico de las piezas de apendicectomía, debido a su baja sospecha diagnóstica clínica.
\end{abstract}

Palabras clave: Tumor carcinoide, apendicectomía, apendicitis aguda.

\section{Aplicación de la microscopia electrónica en el diagnóstico de las patologías renales}

\author{
Nancy Rojas, Nancy Mayo, Elizabeth Neira
} Instituto de Patología, Facultad de Medicina, UNMSM

Objetivos: Detectar, en nuestro medio, en qué nefropatías es imprescindible el uso de la microscopia electrónica. Diseño: Analítico no experimental.

Institución: Instituto de Patología, Facultad de Medicina, UNMSM.

Material biológico: Biopsias renales provenientes del Hospital Cayetano Heredia, cuyo diagnóstico no fue concluyente utilizando las técnicas convencionales hematoxilina eosina, tricrómica de Masson o Mallory, ácido periódico Shiff (PAS), reticulina o inmunofluorescencia.

Intervenciones: Las biopsias fueron procesadas para su estudio ultraestructural con microscopio electrónico de transmisión de UNI, siguiendo los protocolos para el procesamiento de las muestras biológicas.

Principales medidas de resultados: Se observó las características ultraestructurales de los glomérulos, el área mesangial, el endotelio la membrana basal, las células podocitarias, el espacio de Bowman y el epitelio parietal.

Resultados: Estos casos fueron diagnosticados de acuerdo a la clasificación de la Organización Mundial de la Salud y clasificados en glomeruloesclerosis focal y segmentaria, glomerulopatía membranosa estadio I, cambios mínimos.

Conclusiones: El diagnóstico de la mayor parte de las glomerulopatías puede ser realizado mediante técnicas de microscopia de luz e inmunofluorescencia. La microscopia electrónica de transmisión fue esencial para concluir en el diagnóstico de glomerulopatías membranosas y nefropatías hereditarias, como el Síndrome de Alport.

Palabras clave: Glomerulopatías, ultrestructura, microscopía electrónica de transmisión. 